\title{
Identification and analysis of microRNAs in the left ventricular myocardium of two-kidney one-clip hypertensive rats
}

\author{
GANG SUN $^{1}$, HAI HU ${ }^{2}$, XUYANG TIAN ${ }^{1}$, JIANWEI YUE ${ }^{1}$, HUI YU ${ }^{1}$, XIAOMIN YANG ${ }^{1}$ and ZHANLI WANG ${ }^{3}$ \\ ${ }^{1}$ Research Institute of Hypertension, Department of Cardiovascular Medicine, The Second Affiliated Hospital, \\ Baotou Medical College, Baotou, Inner Mongolia Autonomous Region 014030; \\ ${ }^{2}$ Department of Pathophysiology, Baotou Medical College, Baotou, Inner Mongolia Autonomous Region 014060; \\ ${ }^{3}$ Department of Laboratory Centre, The First Affiliated Hospital, Baotou Medical College, \\ Baotou, Inner Mongolia Autonomous Region 014010, P.R. China
}

Received January 30, 2013; Accepted June 11, 2013

DOI: $10.3892 / \mathrm{mmr} .2013 .1549$

\begin{abstract}
MicroRNAs (miRNAs) are involved in the regulation of numerous physiological and pathological processes. However, little information is available with regard to miRNAs in the left ventricular myocardium of the renovascular angiotensin-dependent hypertensive rat. In this study, miRNA expression profiles in the left ventricular myocardium of two-kidney one-clip (2K1C) hypertensive rats were analyzed using a microarray. The roles of the differentially expressed miRNAs, their target genes and signaling pathways were analyzed using ingenuity pathway analysis (IPA) for the first time to further elucidate the molecular mechanisms of left ventricular remodeling.
\end{abstract}

\section{Introduction}

Hypertension is a crucial risk factor for cardiovascular disease. It causes cardiac remodeling, accompanied by fibrosis, inflammation, hypertrophy, pump failure and myocyte degeneration (1). Despite recent advances in the understanding of the molecular and cellular processes of cardiac remodeling, further development of effective therapies for the prevention of heart failure is required.

MicroRNAs (miRNAs) are small, endogenous, non-coding RNAs that have emerged as a new set of modula-

Correspondence to: Dr Zhanli Wang, Department of Laboratory Centre, The First Affiliated Hospital, Baotou Medical College, 41 Linyin Road, Baotou, Inner Mongolia Autonomous Region 014010, P.R. China

E-mail: wang.zhanli@hotmail.com

Dr Xiaomin Yang, Research Institute of Hypertension, Department of Cardiovascular Medicine, The Second Affiliated Hospital, Baotou Medical College, 89 Hude mulin Street, Baotou, Inner Mongolia Autonomous Region 014030, P.R. China

E-mail: yangxm209@163.com

Key words: hypertension, left ventricular remodeling, microRNA, microarray, expression profile tors of gene expression (2). It is estimated that $>1,000$ unique miRNAs are encoded within the human genome (3). These miRNAs bind with imperfect complementarity to their target mRNAs and regulate a variety of diseases, such as cancer, Alzheimer's disease and cardiovascular diseases (4-6). Recent studies have revealed that miRNAs modulate the pathogenesis of cardiac remodeling (7). The expression profile of miRNAs in failing myocardium has been explored by performing microarrays on various murine models of cardiac hypertrophy, including calcineurin overexpression (6), Akt overexpression (8), coronary artery ligation, ischemia-reperfusion (9) and thoracic aortic constriction (10). Based on these murine models, a variety of miRNAs involved in cardiac myocyte hypertrophy have been identified (11-14). However, little information is available with regard to miRNAs in the renovascular hypertensive murine model. The roles and expression profiles of miRNAs in the left ventricular myocardium of renovascular hypertensive rats are poorly understood.

This study aims to investigate the miRNA expression profile associated with left ventricular remodeling in two-kidney one-clip (2K1C) hypertensive rats. Target genes and biological networks of the differentially expressed miRNAs were also identified. Furthermore, the possible mechanisms of miRNA-mediated signaling were analyzed using ingenuity pathway analysis (IPA) for the first time.

\section{Materials and methods}

Animals. Male Wistar rats (weight, 120-150 g), purchased from Vital River Lab Animal Technology Co., Ltd. (Beijing, China), were housed individually in temperature- $\left(20 \pm 2^{\circ} \mathrm{C}\right)$ and humidity $(55 \pm 5 \%)$-controlled rooms, with a 12 -hour light/dark cycle. The rats were given free access to food and tap water. Animal studies were performed under conditions approved by the Local Animal Care and Use Committee.

Induction of 2 K1C hypertension. Following one week of acclimatization, the rats were randomly divided into two groups. As described in detail previously (15), the male Wistar rats $(n=14)$ were anesthetized with sodium 
Table I. Comparison of organ indices in sham surgery $(\mathrm{n}=12)$ and $2 \mathrm{~K} 1 \mathrm{C}$ rats $(\mathrm{n}=14)$.

\begin{tabular}{lccc}
\hline Group & $\begin{array}{c}\text { Heart } \\
\text { weight }(\mathrm{g})\end{array}$ & $\begin{array}{c}\text { Cardiac } \\
\text { index }\end{array}$ & $\begin{array}{c}\text { Right renal } \\
\text { index }\end{array}$ \\
\hline Sham surgery & $1.08 \pm 0.06$ & $(2.8 \pm 0.3) \times 10^{-3}$ & $(3.3 \pm 0.5) \times 10^{-3}$ \\
2K1C & $1.20 \pm 0.14^{\mathrm{a}}$ & $(3.2 \pm 0.3) \times 10^{-3 \mathrm{a}}$ & $(4.9 \pm 1.1) \times 10^{-3 \mathrm{a}}$
\end{tabular}

${ }^{\text {a }} \mathrm{P}<0.01$ vs. the sham-surgery group. Results are presented as the mean \pm SEM. 2K1C, two-kidney one-clip.

pentobarbital $(50 \mathrm{mg} / \mathrm{kg}$ body weight, i.p.) and the left kidney was exposed by a flank incision. After separating the renal arteries, a silver clip was placed around the left renal artery. Sham surgery rats $(n=12)$ underwent an identical surgical procedure with the exception of the placing of the arterial clip. The systolic blood pressure (SBP) of the rats in each group was measured weekly using a non-invasive computerized tail-cuff system (HX-II, Hunan Medical Univ. Medical Instruments Inc., Changsha, China). Eight weeks after the initial surgery, the rats were anesthetized with sodium pentobarbital following overnight fasting. Organs of interest (the heart and kidneys) were immediately removed, blotted dry, weighed and stored at $-80^{\circ} \mathrm{C}$ until RNA extraction. Organ indices were calculated using the following formula: organ index $=$ organ weight $(\mathrm{g}) /$ body weight $(\mathrm{g}) \times 100$.

Histologic analysis. Heart samples from each rat were fixed in $10 \%$ buffered formalin and embedded in paraffin. Heart sections of 5-6 $\mu \mathrm{m}$ were cut and stained with hematoxylin-eosin (HE) and van Gieson's (VG). Histopathological changes were examined under the microscope.

RNA isolation and miRNA microarray. Total RNA was extracted from $30 \mathrm{mg}$ of tissues. Samples were mechanically disrupted and simultaneously homogenized in the presence of QIAzol Lysis reagent (Qiagen, Valencia, CA, USA) using a Mikro-Dismembrator (Braun Biotech International, Melsungen, Germany). RNA was extracted using the miRNeasy Mini kit (Qiagen) according to the manufacturer's instructions. RNA concentrations were measured using the NanoDrop 2000c Spectrophotometer (NanoDrop Technologies, Wilmington, DE, USA), while RNA quality was assessed using the Agilent 2100 Bioanalyzer (Agilent Technologies, Santa Clara, CA, USA) using the RNA 6000 Nano kit (Agilent Technologies). Total RNA (100 ng) was dephosphorylated at $37^{\circ} \mathrm{C}$ for $30 \mathrm{~min}$ with calf intestinal phosphatase and denatured using $100 \% \mathrm{DMSO}$ at $100^{\circ} \mathrm{C}$ for 5 min. Samples were labeled with Cyanine 3-pCp using T4 DNA ligase (Invitrogen, Grand Island, NY, USA) by incubation at $16^{\circ} \mathrm{C}$ for $1 \mathrm{~h}$ and by hybridization on a $8 \times 15 \mathrm{~K}$ format Agilent rat miRNA array G2534A (Agilent Technologies). Arrays were washed according to the manufacturer's instructions and scanned at a resolution of $5 \mu \mathrm{m}$ using an Agilent G2565CA microarray scanner (Agilent Technologies). Data were acquired and the quantile was normalized using Agilent Feature Extraction software version 9.5.3 (Agilent Technologies).
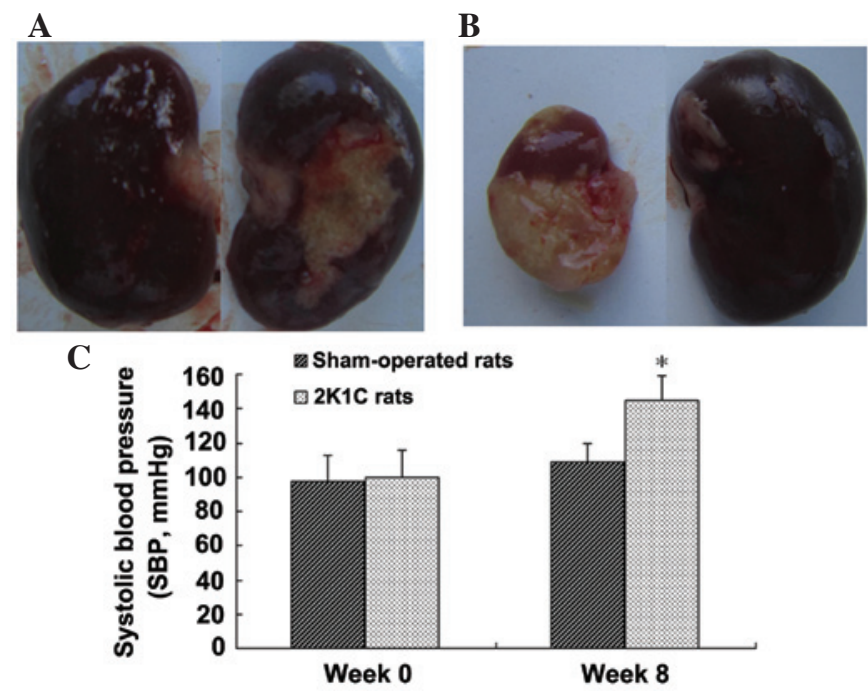

Figure 1. (A) Left (left) and right kidneys (right) of a sham surgery rat, eight weeks after surgery. (B) The clipped kidney (left) and the non-clipped kidney (right) of a 2K1C rat, eight weeks after clipping. (C) Systolic blood pressure in the $2 \mathrm{~K} 1 \mathrm{C}$ rats compared with the sham surgery rats. ${ }^{*} \mathrm{P}<0.01$ vs. the sham surgery group. $2 \mathrm{~K} 1 \mathrm{C}$, two-kidney one-clip.

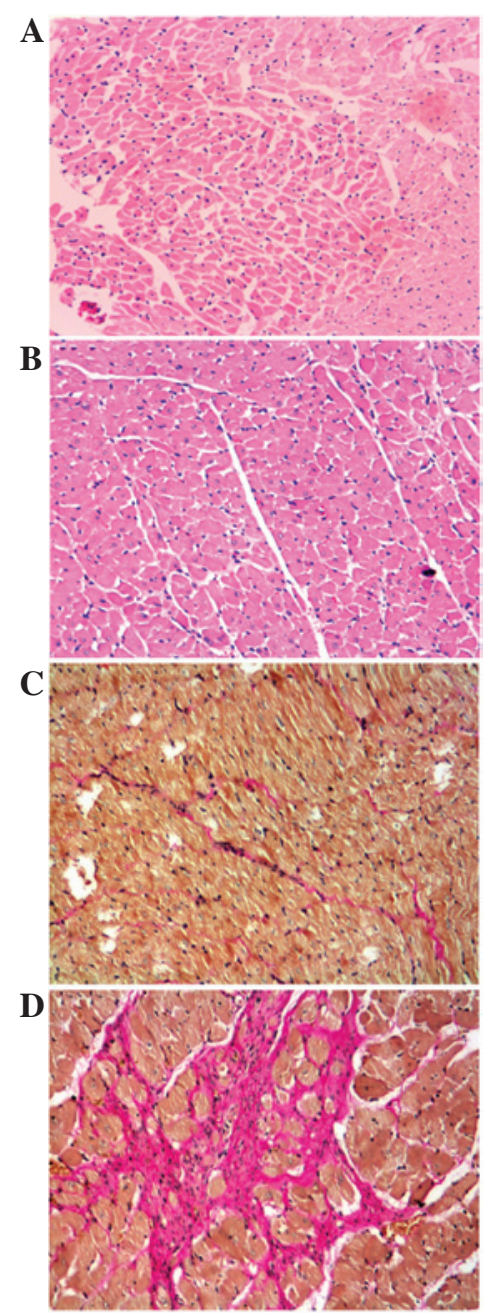

Figure 2. Cardiomyoctyes stained with hematoxylin-eosin (HE) and myocardial interstitial collagen deposition stained with van Gieson's (VG). (A) Size of cardiomyoctyes in sham surgery rats. (B) Size of cardiomyoctyes in 2K1C rats. (C) Myocardial interstitial collagen deposition in sham surgery rats. (D) Myocardial interstitial collagen deposition in $2 \mathrm{~K} 1 \mathrm{C}$ rats. 2K1C, two-kidney one-clip. 


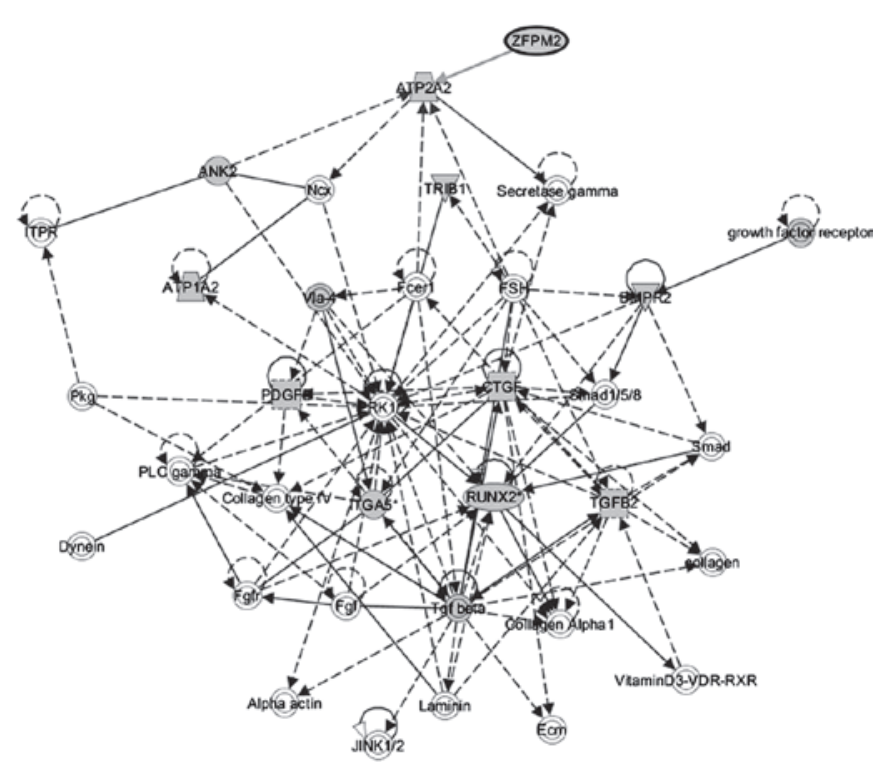

Figure 3. Representation of the most highly rated network (cardiovascular disease, cardiovascular system development and function, organ morphology). Molecules are represented as nodes. The molecules shaded gray represent the target genes of differentially expressed miRNAs. A solid line represents a direct interaction between two gene products and a dotted line represents an indirect interaction.

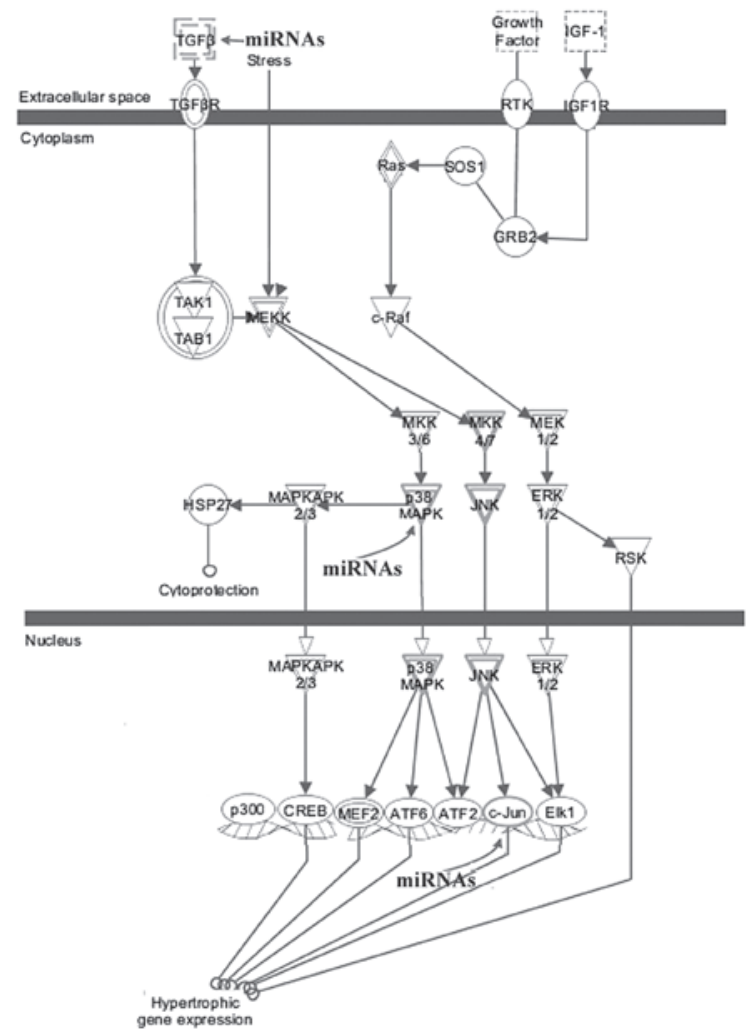

Figure 4. Cardiac hypertrophy signaling pathway obtained from IPA analysis. IPA, ingenuity pathway analysis.

Bioinformatics analysis. Datasets representing miRNAs with altered expression profiles derived from microarray analyses were imported into the IPA program (http://www.ingenuity.com). The basis of the IPA tool consists of the ingenuity pathway knowledge base (IPKB) derived from
Table II. Differentially expressed miRNAs in $2 \mathrm{~K} 1 \mathrm{C}$ rats.

Downregulated expression

\begin{tabular}{lr} 
Systematic name & Fold change \\
\hline rno-miR-139-5p & -38.4905200 \\
rno-miR-224 & -26.9225270 \\
rno-miR-129-2* & -17.7938580 \\
rno-miR-129-1* & -17.4628870 \\
rno-miR-92b & -15.4211950 \\
rno-miR-505* & -10.1010240 \\
rno-miR-127 & -6.6837770 \\
rno-miR-141 & -4.8718386 \\
rno-miR-224* & -4.8661530 \\
rno-miR-455 & -4.3821400 \\
rno-miR-384-5p & -4.2651870 \\
rno-miR-362 & -3.9236674 \\
rno-miR-122 & -3.6222723 \\
rno-miR-363 & -3.3113322 \\
rno-miR-434 & -3.2692702 \\
rno-miR-425* & -3.1358542 \\
rno-miR-872* & -3.1162152 \\
rno-miR-582 & -3.0495758 \\
rno-miR-667 & -2.9269574 \\
rno-miR-547* & -2.8845766 \\
rno-miR-483 & -2.8532393 \\
rno-miR-1188-3p & -2.1270647 \\
rno-miR-10b & -2.0181155 \\
\hline
\end{tabular}

Upregulated expression

Systematic name

Fold change

rno-miR-3544

$+2.0963676$

rno-miR-298

$+2.1669054$

rno-miR-196c

$+2.2632172$

rno-miR-181a- $1 *$

$+2.3843806$

rno-miR-598-3p

$+2.3982275$

rno-miR-188

$+2.4536152$

rno-miR-760-5p

$+2.4810490$

rno-miR-335

$+2.4852781$

rno-miR-31

$+2.7537080$

rno-miR-190*

$+2.7569172$

rno-miR-484

$+2.7729888$

rno-miR-672

rno-miR-874

rno-miR-455*

$+2.8125286$

$+2.8486352$

$+2.8730786$

$+3.1480370$

rno-miR-3584-5p

$+3.5094187$

rno-miR-132

rno-miR-345-3p

$+3.6336637$

rno-miR-136

$+3.6917052$

rno-miR-3588

$+3.7029164$

rno-miR-31*

$+3.7426915$

rno-miR-20a*

$+5.3322973$

rno-miR-32*

rno-miR-3562

$+6.2950974$

$+9.9319600$

rno-miR-338*

$+18.3044530$

rno-miR-139-3p

$+49.2387500$

2K1C, two-kidney one-clip. Asterisk indicates the less predominant form of the miRNA arising from the opposite arm of a shared pre-miRNA hairpin. 
Table III. Target genes for differentially expressed miRNAs in $2 \mathrm{~K} 1 \mathrm{C}$ rats.

\begin{tabular}{|c|c|c|c|c|c|c|c|}
\hline \multicolumn{8}{|c|}{ Target genes } \\
\hline$A B L 1$ & $A D A M 17$ & AKT3 & $A L O X 5 A P$ & ANK2 & ANXAl & AP3M2 & $A T P 1 A 2$ \\
\hline$A T P 2 A 2$ & BCL2L11 & BCL6* & BECN1 & $B M P R 2$ & $C D C P 1$ & $C D K N 1 A$ & $C D K N 2 A$ \\
\hline CERS6 & $C T G F$ & CTNNBI & EGLN3 & $F 2$ & $F B X W 7$ & FOXP1 & FOXP3 \\
\hline GEMIN2 & GNAI2 & HIF $1 A$ & $I D H I$ & $I K B K B$ & ILII & $I T G A 5^{*}$ & $J A K 2$ \\
\hline$J U N$ & $M A P 2 K 4^{*}$ & P38MAPK & MMP9 & NCEH1 & NFATC1 & $N P R 3$ & NT5E \\
\hline NTRK3 & $P D G F B$ & РPРЗСА & PTEN & $R B 1$ & RHOA & $R U N X 2 *$ & S100A9 \\
\hline SOCS3 & STX7 & $T G F B 2$ & TP53 & TRIBI & VSNL1 & $X B P 1$ & ZFPM2 \\
\hline
\end{tabular}

$2 \mathrm{~K} 1 \mathrm{C}$, two-kidney one-clip. Asterisk follows the locus designation and precedes the allele designation if there is one. For example, BCL6 ${ }^{*}$ is the symbol for the gene locus that encodes B-cell lymphoma 6 protein BCL6.

Table IV. Top five networks with their respective scores obtained from IPA.

\begin{tabular}{lll} 
ID & \multicolumn{1}{c}{ Associated network functions } & Score \\
\hline 1 & Cardiovascular disease, cardiovascular system development and function, organ morphology & 22 \\
2 & Cancer, hematological disease, neurological disease & 15 \\
3 & Cellular development, cellular growth and proliferation, hematological system development and function & 13 \\
4 & Cell death and survival, cancer, hematological disease & 12 \\
5 & Hematological system development and function, tissue morphology, organismal injury and abnormalities & 10 \\
\hline
\end{tabular}

IPA, ingenuity pathway analysis.

Table V. Top five toxicology pathways obtained from IPA.

\begin{tabular}{llr}
\hline Pathway & P-value & \multicolumn{1}{c}{ Ratio } \\
\hline Cardiac hypertrophy & $6.39 \times 10^{-19}$ & $19 / 333(0.057)$ \\
Cardiac necrosis/cell death & $1.71 \times 10^{-14}$ & $14 / 227(0.062)$ \\
Liver proliferation & $5.38 \times 10^{-11}$ & $11 / 201(0.032)$ \\
Renal necrosis/cell death & $1.21 \times 10^{-10}$ & $14 / 437(0.032)$ \\
P53 signaling & $9.18 \times 10^{-10}$ & $8 / 95(0.084)$
\end{tabular}

IPA, ingenuity pathway analysis.

the known functions and interactions of miRNAs published in the literature. In this study, the target genes, biological networks and functional pathways of the differentially expressed miRNAs were identified.

Statistical analysis. Statistical analysis was performed using SPSS 17.0 (SPSS Inc., Somers, New York, USA). The differences between groups were compared using the Student's t-test. Correlations among the expression profiles of miRNAs were calculated using Pearson's correlation. $\mathrm{P}<0.05$ was considered to indicate a statistically significant difference. All values are expressed as the mean \pm SEM.

\section{Results}

Establishment of $2 \mathrm{~K} 1 \mathrm{C}$ hypertensive rats. We developed a rat model of $2 \mathrm{~K} 1 \mathrm{C}$ hypertension as previously described (15). Eight weeks after renal artery clipping, the $2 \mathrm{~K} 1 \mathrm{C}$ rats

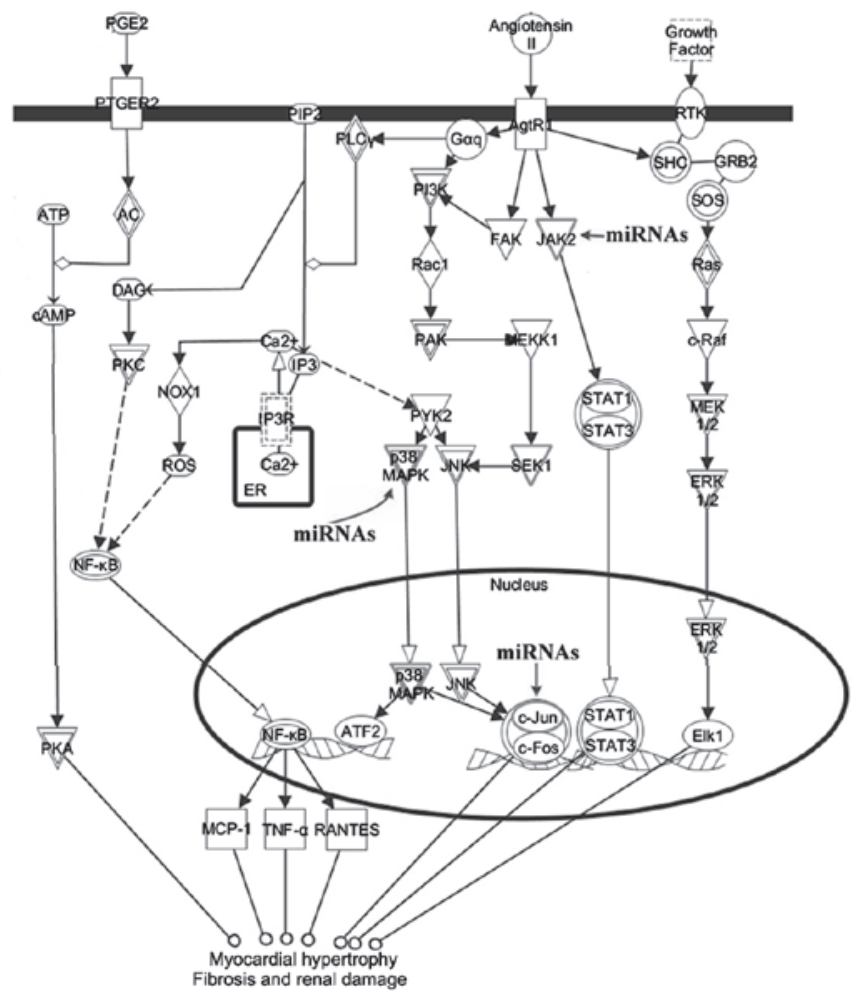

Figure 5. Renin-angiotensin signaling pathway obtained from IPA analysis. IPA, ingenuity pathway analysis.

exhibited atrophy of the clipped (left) kidney and hypertrophy of the non-clipped (right) kidney $(\mathrm{P}<0.05$; Fig. 1$) .2 \mathrm{~K} 1 \mathrm{C}$ rats 
exhibited an increase in SBP $(\mathrm{P}<0.05)$, while the SBP of the sham surgery rats remained unaltered (Fig. 1). Furthermore, $2 \mathrm{~K} 1 \mathrm{C}$ caused a significant increase in the weight of the heart, the cardiac index and the right renal index when compared with the sham surgery group $(\mathrm{P}<0.05$; Table I). Additionally, our results demonstrated that $2 \mathrm{~K} 1 \mathrm{C}$ promoted cardiomyocyte hypertrophy and increased interstitial collagen deposition and infiltration of the myocardium (Fig. 2).

Identification of the miRNA expression profile. In order to identify differentially expressed miRNAs in $2 \mathrm{~K} 1 \mathrm{C}$ rats, we performed miRNA microarray analyses. Of the miRNAs investigated, our results identified 48 that were differentially expressed between the sham surgery group and the $2 \mathrm{~K} 1 \mathrm{C}$ group, of which 25 were upregulated and 23 were downregulated in the left ventricular myocardium of the $2 \mathrm{~K} 1 \mathrm{C}$ hypertensive rats (Table II). A 2.0-fold change cut-off was applied to all array data sets.

The target genes and biological networks of differentially expressed miRNAs. The target genes of differentially expressed miRNAs were analyzed using the IPA tool, which combined the sources of TarBase, TargetScan, miRecords and Ingenuity Expert. A total of 10,156 target genes were identified; of these, 1,140 presented in the rats. In order to eliminate the false positive rates of the target prediction databases, only the target genes validated by biological experiments were selected for further analysis. Following the analysis of published data, 56 target genes were identified (Table III). Highly rated networks of 56 target genes were analyzed and the results revealed five significant pathways (Table IV). Of these networks, 'cardiovascular disease, cardiovascular system development and function, organ morphology' was the highest rated network with a significance score of 22. Target genes ANK2, ATP1A2, ATP1A2, BMPR2, CTGF, ITGA5*, PDGFB, $R U N X 2 *, T G F B 2, T R I B 1$ and ZFPM2 were involved in this network (Fig. 3). Moreover, the genes associated with the top toxicology list are provided in Table V. 'Cardiac hypertrophy' and 'cardiac necrosis/cell death' were identified as the top two toxicology pathways.

Potential mechanisms of the differentially expressed miRNAs in the development of left ventricular remodeling. Signal transduction pathways associated with target genes of the differentially expressed miRNAs were investigated. IPA analysis revealed that the 56 target genes participated in pathways linked to cardiac hypertrophy (Fig. 4). Certain target genes, including TGF $\beta, p 38 M A P K$ and $c$-Jun, were involved in the cardiac hypertrophy signaling pathway. We also examined the roles of the target genes as mediators of renin-angiotensin signaling. We discovered that p38MAPK, c-Jun and JAK2 were involved in renin-angiotensin signaling in response to left ventricular remodeling (Fig. 5).

\section{Discussion}

The $2 \mathrm{~K} 1 \mathrm{C}$ rat model is useful for studying the molecular mechanisms involved in hypertension-related cardiac remodeling. In an attempt to identify the expression profiles of the miRNAs in this experimental model, we undertook systematic profiling using a commercially available microarray platform. We discovered that $48 \mathrm{miRNAs}$ were differentially expressed between the $2 \mathrm{~K} 1 \mathrm{C}$ and sham surgery groups. Notably, cardiac remodeling represents collective changes in the biology of the cardiac myocyte, the volume of myocyte and non-myocyte components of the myocardium, in addition to the geometry of the left ventricular chamber. Therefore, the altered miRNA levels should be attributed to cardiomyocytes in addition to other myocardial cell types, such as fibroblasts and endothelial cells.

To understand the involvement of miRNAs in left ventricular remodeling, we selected differentially expressed miRNAs and performed target gene analysis. Due to the complex nature of cardiac remodeling and since existing computational algorithms remain challenging, the target prediction analysis was unable to reveal specific sets of genes involved in defined biological aspects. Thus, only 56 target genes that had been validated by biological experiments were selected for further analysis in the current study. Moreover, IPA analysis revealed 'cardiovascular disease, cardiovascular system development and function, organ morphology' to be the most favored associated network function in $2 \mathrm{~K} 1 \mathrm{C}$ rats. The two most significant pathways 'cardiac hypertrophy' and 'cardiac necrosis/cell death' were observed. These results suggested that alterations to the expression of miRNAs observed in this study represented the physiological responses to an altered cardiac workload.

To gain an understanding of the molecular mechanisms of left ventricular remodeling, signal transduction pathways associated with the differentially expressed miRNAs and their target genes were investigated. This study revealed that the target genes participated in pathways linked to cardiac hypertrophy. Signaling molecules, such as TGF $\beta$ and p38MAPK, were validated target genes. Our results suggested that differentially expressed miRNAs were mediators of the TGF $\beta$-TAK1-p38MAPK signaling pathway, which is crucial for myocyte hypertrophy, as described previously (16). Renovascular hypertensive rats were used in this experiment. The renin-angiotensin system contributes to left ventricular hypertrophy and fibrosis (17). Therefore, the correlations between the renin-angiotensin signaling pathway and the target genes of differentially expressed miRNAs were evaluated. In addition to a p38MAPK-dependent pathway contributing to left ventricular hypertrophy, we observed that the target gene JAK2 is a signaling molecule, suggesting that the differentially expressed miRNAs were also sensitive to the JAK2-STAT signaling pathway, which is crucial for cardiac fibrosis (18).

In conclusion, the miRNA expression profiles in the left ventricular myocardium of $2 \mathrm{~K} 1 \mathrm{C}$ hypertensive rats were identified. Further analysis of the roles of the differentially expressed miRNAs, their target genes and signaling pathways would provide greater insight into the molecular mechanisms of left ventricular remodeling.

\section{Acknowledgements}

This work was supported by the National Natural Science Foundation of China (no. 81160033). We thank Mr. J. C. Lin of CloudScientific Technology Co., Ltd. for performing the ingenuity pathway analyses. 


\section{References}

1. Soares ER, Lima WG, Machado RP, et al: Cardiac and renal effects induced by different exercise workloads in renovascular hypertensive rats. Braz J Med Biol Res 44: 573-582, 2011.

2. Lecellier CH, Dunoyer P, Arar K, et al: A cellular microRNA mediates antiviral defense in human cells. Science 308: 557-560, 2005.

3. Berezikov E, Guryev V, van de Belt J, et al: Phylogenetic shadowing and computational identification of human microRNA genes. Cell 120: 21-24, 2005.

4. Jost D, Nowojewski A and Levine E: Small RNA biology is systems biology. BMB Rep 44: 11-21, 2011.

5. Lukiw WJ: Micro-RNA speciation in fetal, adult and Alzheimer's disease hippocampus. Neuroreport 18: 297-300, 2007.

6. van Rooij E, Sutherland LB, Liu N, et al: A signature pattern of stress-responsive microRNAs that can evoke cardiac hypertrophy and heart failure. Proc Natl Acad Sci USA 103: 18255-18260, 2006.

7. Yang B, Lin H, Xiao J, et al: The muscle-specific microRNA miR-1 regulates cardiac arrhythmogenic potential by targeting GJA1 and KCNJ2. Nat Med 13: 486-491, 2007.

8. Carè A, Catalucci D, Felicetti F, et al: MicroRNA-133 controls cardiac hypertrophy. Nat Med 13: 613-618, 2007.

9. van Rooij E, Sutherland LB, Thatcher JE, et al: Dysregulation of microRNAs after myocardial infarction reveals a role of miR-29 in cardiac fibrosis. Proc Natl Acad Sci USA 105: 13027-13032, 2008.

10. Sayed D, Hong C, Chen IY, Lypowy J and Abdellatif M MicroRNAs play an essential role in the development of cardiac hypertrophy. Circ Res 100: 416-424, 2007.
11. Elia L, Contu R, Quintavalle M, et al: Reciprocal regulation of microRNA-1 and insulin-like growth factor-1 signal transduction cascade in cardiac and skeletal muscle in physiological and pathological conditions. Circulation 120: 2377-2385, 2009.

12. Horie T, Ono K, Nishi H, et al: MicroRNA-133 regulates the expression of GLUT4 by targeting KLF15 and is involved in metabolic control in cardiac myocytes. Biochem Biophys Res Commun 389: 315-320, 2009.

13. Thum T, Gross C, Fiedler J, et al: MicroRNA-21 contributes to myocardial disease by stimulating MAP kinase signalling in fibroblasts. Nature 456: 980-984, 2008.

14. Lin Z, Murtaza I, Wang K, Jiao J, Gao J and Li PF: miR-23a functions downstream of NFATc3 to regulate cardiac hypertrophy. Proc Natl Acad Sci USA 106: 12103-12108, 2009.

15. Wiesel P, Mazzolai L, Nussberger $J$ and Pedrazzini $T$ : Two-kidney, one clip and one-kidney, one clip hypertension in mice. Hypertension 29: 1025-1030, 1997.

16. Matsumoto-Ida M, Takimoto Y, Aoyama T, Akao M, Takeda T and Kita T: Activation of TGF-beta1-TAK1-p38 MAPK pathway in spared cardiomyocytes is involved in left ventricular remodeling after myocardial infarction in rats. Am J Physiol Heart Circ Physiol 290: H709-H715, 2006.

17. Mascareno E, Galatioto J, Rozenberg I, et al: Cardiac lineage protein-1 (CLP-1) regulates cardiac remodeling via transcriptional modulation of diverse hypertrophic and fibrotic responses and angiotensin II-transforming growth factor $\beta$ (TGF- $\beta 1$ ) signaling axis. J Biol Chem 287: 13084-13093, 2012.

18. Liao W, Yu C, Wen J, et al: Adiponectin induces interleukin-6 production and activates STAT3 in adult mouse cardiac fibroblasts. Biol Cell 101: 263-272, 2009. 